Classification

Physics Abstracts

82.80-d

\title{
Comparison of detection limits for EELS and EDXS
}

\author{
Richard D. Leapman $\left({ }^{1}\right)$ and John A. Hunt $\left({ }^{2}\right)$ \\ ( $\left.{ }^{1}\right)$ Biomedical Engineering and Instrumentation Program, NCRR, National Institutes of Health, \\ Bethesda, Maryland 20892, U.S.A. \\ (2) Department of Materials Science and Engineering, Lehigh University, Bethlehem, Pennsylvania \\ 18015-3195, U.S.A.
}

(Received November 21, 1990; accepted January 28, 1991)

\begin{abstract}
Simultaneous measurements have been made in the scanning transmission electron microscope to determine relative microanalytical sensitivities for electron energy loss (EELS) and energy-dispersive x-ray spectroscopies (EDXS). A photodiode-array parallel detector was used for EELS, and an ultrathin window $\mathrm{Si}(\mathrm{Li})$ detector subtending a solid angle of 0.18 sterad was used for EDXS. Energy loss spectra were acquired in the first or second difference mode to reduce channelto-channel gain variations and to detect weak signals on large backgrounds. Both EELS and EDXS data were analyzed using multiple least squares procedures to fit reference spectra; this gave not only the fitting coefficients but also their standard deviations. Results showed that EELS is the method of choice for analysis of elements with $Z<10$. For higher atomic numbers $(10<Z<25)$ EDXS is preferable to EELS if the K edge is used. However, EELS has the higher sensitivity for most elements in this atomic number range if the $\mathrm{L}_{23}$ edge is analyzed. For sodium detection limits are approximately a factor of 10 better with EELS. As the atomic number increases this factor becomes smaller although EELS still has a significant advantage for phosphorus. The occurence of the "white-line" resonance at the $\mathrm{L}_{23}$ edge for $Z>20$ boosts the advantage of EELS for calcium but at iron EELS and EDXS have about the same sensitivity. Calculated estimates of the relative sensitivities for EELS and EDXS are in reasonable agreement with the experimental data.
\end{abstract}

\section{Introduction.}

Microanalytical sensitivity of electron energy loss spectroscopy (EELS) has been greatly increased in recent years by using electronic multichannel arrays to detect in parallel the inelastically scattered electrons [1,2]. At the same time improvements have been made in the sensitivity of energydispersive $\mathrm{x}$-ray spectroscopy (EDXS) through increasing the geometric collection efficiency of the detector crystal and by utilizing an ultra-thin window (UTW) or by completely removing the window [3]. These developments now make it more difficult to decide whether EELS or EDXS will yield the better detection limits for microanalysis of a specific element in the scanning transmission electron microscope (STEM). Originally it was believed that EELS should provide higher sensitivity for light elements, e.g. the first row of the periodic table, because of the large K-shell ionization cross section, the low fluorescence yield for $\mathrm{x}$-ray production, and the strong absorption of soft x-rays by the detector window. On the other hand it was believed that EDXS offered better 
detectability for heavier atoms because of the higher $\mathrm{x}$-ray yield and poorer signal/background in the energy loss spectrum. More recently it has been demonstrated, however, that light atoms can be detected by UTW-EDXS [4] and that elements as heavy as uranium can be detected easily by parallel detection EELS [5]. In this paper we therefore attempt to readdress the question originally posed by Isaacson and Johnson [6] as to the relative sensitivities of EELS and EDXS. We shall confine our discussion to samples containing dilute concentrations of elements with $Z<30$ in a thin carbon matrix with a total thickness less than the inelastic mean free path (approximately $60 \mathrm{~nm}$ at $100 \mathrm{keV}$ beam energy). Although this composition relates directly to organic, biological or polymer samples, the same approach could be used to compare EELS and EDXS sensitivities for detecting low elemental concentrations in metals, semiconductors, ceramics or other materials.

\section{Experimental methods.}

Measurements of the relative sensitivities for microanalysis by EELS and EDXS were made using a VG Microscopes HB501 field-emission STEM. The microscope was equipped with a Gatan parallel-detection magnetic sector spectrometer (model 666) [7], and a Tracor Northern UTW silicon x-ray detector (Micro-ZHV Series) [8]. In order to record EELS and EDXS spectra simultaneously a $25 \mu \mathrm{m}$ "virtual" rather than "real" objective aperture was selected to define the probe on the sample and avoid spurious $\mathrm{x}$-ray generation.

The electron spectrometer design had been specially modified for ultrahigh vacuum compatibility to allow baking of the microscope column [7]. A series of quadrupole lenses permitted adjustment of the dispersion so that the energy per channel could be varied. The 1024-channel photodiode array detector was read out in $25 \mathrm{~ms}$ and integration times could be varied from $25 \mathrm{~ms}$ to several seconds. For electron fluxes greater than $10^{3}$ per diode element the photodiode array has been shown to have a detective quantum efficiency (DQE) close to unity [2]. Precise energy offsets could be given to the spectrum by applying a voltage to the electrically isolated drift tube. The narrow intrinsic energy spread of the cold field-emission gun source provided an effective energy resolution of about $0.4 \mathrm{eV}$. The spectrometer acceptance semi-angle was approximately $20 \mathrm{mrad}$ as defined by a $4 \mathrm{~mm}$ aperture in the annular dark-field detector. Spectra were acquired using the Gatan EL/P program on an Apple Macintosh 2 computer [9] and were transferred to a PC/AT- compatible computer [10] for processing.

The active area of the $\mathrm{Si}(\mathrm{Li}) \mathrm{x}$-ray detector was $30 \mathrm{~mm}^{2}$ and was situated at a mean distance of $13 \mathrm{~mm}$ from the sample with a solid angle of $\sim 0.18$ sterad. The detector window consisted of $100 \mathrm{~nm}$ of parylene covered by $15 \mathrm{~nm}$ of aluminium as a light shield. A detector resolution of $\sim 150 \mathrm{eV}$ at the $\mathrm{Mn} \mathrm{K}_{\alpha}$ line was achievable. X-ray spectra were acquired and processed using a Tracor Northern TN5500 computer system.

Specimens were prepared by depositing solutions of compounds on to thin $(\sim 10 \mathrm{~nm})$ carbon films supported on copper grids and by subsequently air-drying the grids. The deposited elements were sodium and chlorine $(\mathrm{NaCl}$ in water $)$, phosphorus $\left(\mathrm{NaH}_{2} \mathrm{PO}_{4}\right.$ in water $)$, and fluorine (octafluorostilbene).

\section{Estimated relative sensitivities for EELS and EDXS.}

It is useful to obtain simple expressions for the ratio of the characteristic energy loss and $x$-ray signals and the ratios of their relative microanalytical sensitivities. We can then compare these values with statistical variations found in simultaneously measured EELS and EDXS spectra. The 
derived expressions also indicate systematic trends in relative sensitivities as a function of atomic number.

The ratio of signals for EELS and EDXS can be written approximately as,

$$
S_{\mathrm{E}} / S_{\mathrm{X}}=\left(Q_{\mathrm{E}} / Q_{\mathrm{X}}\right)\left(\eta_{\mathrm{E}} / \eta_{\mathrm{X}}\right) f_{\mathrm{E}} \omega_{\mathrm{X}}^{-1} \exp \left(-t / \lambda_{\mathrm{i}}\right) \exp \left(\mu_{\mathrm{X}} d\right)
$$

where, E denotes EELS and X denotes EDXS, $Q$ is the total ionization cross section, $\eta$ is the geometric collection efficiency of the signal. $\omega_{\mathrm{X}}$ is the X-ray fluorescence yield which falls off rapidly with decreasing atomic number. $f_{\mathrm{E}}$ is the fraction of the total core edge intensity that can be utilized for microanalysis. Conventionally a large energy window, typically 50 or $100 \mathrm{eV}$, is chosen to perform EELS microanalysis and this is reasonable when the background can be subtracted satisfactorily. In this work, we make an important distinction for the situation where the signal is very small compared to the background, and where small errors in the background estimation cause relatively large errors in the signal. Under these conditions it is advantageous to choose a narrower energy window, even, though $f_{\mathrm{E}}$ is significantly reduced. The remaining factors in equation (1) involve attenuation of the EELS signal by plural scattering in a sample of thickness $t$ having an inelastic mean free path $\lambda_{\mathrm{i}}$, and attenuation of the X-ray signal by the detector window of effective thickness $d$ having absorption coefficient $\mu_{\mathrm{X}}$. Both of these exponential factors in equation (1) will be neglected here because the samples are thin and a UTW X-ray detector is used.

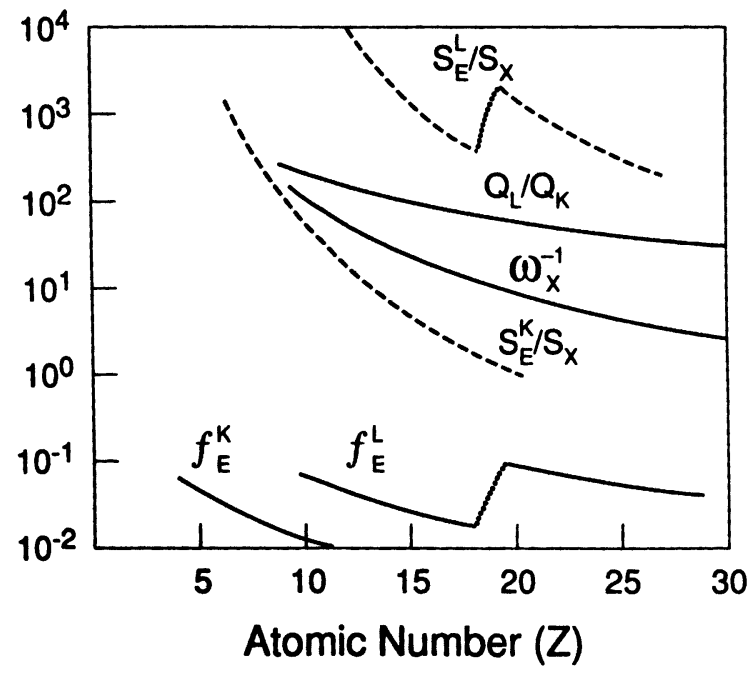

Fig. 1. - Estimated ratios of $S_{\mathrm{E}} / S_{\mathrm{X}}$ as a function of atomic number $(Z<30)$ for $\mathrm{K}$ and $\mathrm{L}_{23}$ edges (dashed lines). Other parameters in equation 1 are also plotted: ratio of $\mathrm{K}$ and $\mathrm{L}_{23}$ cross sections $\left(Q_{\mathrm{L}} / Q_{\mathrm{K}}\right)$, inverse fluorescence yield $\left(\omega_{\mathrm{X}}^{-1}\right)$, and fraction of core edge cross section in edge region $\left(f_{\mathrm{E}}(\Delta, \beta)\right)$ (solid lines).

Estimated ratios of $S_{\mathrm{E}} / S_{\mathrm{X}}$ are plotted in figure 1 as a function of atomic number $(Z<30)$ for both the $\mathrm{K}$ and $\mathrm{L}_{23}$ shell excitations in the energy loss spectrum and for the $K_{\alpha}$ emission line in the $\mathrm{X}$-ray spectrum. In addition several of the parameters in equation (1) are also plotted: $f_{\mathrm{E}}(\Delta, \beta)$, the fraction of the total core edge cross section in the near-edge region of energy width $\Delta$ and containing scattering semi-angles $\beta$ (defined by the spectrometer entrance aperture); $Q_{\mathrm{L}} / Q_{\mathrm{K}}$, 
the ratio of the $\mathrm{K}$ and $\mathrm{L}_{23}$ cross sections; and $\omega_{\mathrm{X}}^{-1}$, the inverse of the fluorescence yield. Ratios of total cross sections, $Q_{\mathrm{L}} / Q_{\mathrm{K}}$, were obtained from the SIGMAK and SIGMAL programs of Egerton [11], and $\omega_{\mathrm{X}}^{-1}$ was calculated from Heinrich [12]. The parameters used to calculate $S_{\mathrm{E}} / S_{\mathrm{X}}$ are also listed in table I for the elements that we consider in this paper.

Table I. - Parameters used in equation (1) to predict ratios of EELS and EDXS signals, for elements $F, \mathrm{Na}, \mathrm{P}$ and $\mathrm{Cl}$ at $100 \mathrm{keV}$ beam energy. Parallel detection EELS with DQE $=1$ and collection semiangle $\beta=20 \mathrm{mrad}$ were assumed. Energy window $\Delta$ was chosen to correspond to first or second difference filter width, and $f_{\mathrm{E}}(\Delta, \beta)$ was estimated from cross section data [11]. $Q_{\mathrm{E}}$ and $Q_{\mathrm{X}}$ are total ionization cross sections for atomic shells producing EELS and EDXS signals respectively. The $U T W$-EDXS detector was assumed to subtend solid angle of 0.18 sterad, giving $\eta_{\mathrm{E}} / \eta_{\mathrm{X}}=70$; only the $K_{\alpha}$ line was considered and absorption of $x$-rays was neglected.

\begin{tabular}{|l|r|r|r|r|r|r|}
\hline Element/ & $Z$ & Energy & $\Delta$ & $f_{\mathrm{E}}(\Delta, \beta)$ & $\omega_{\mathrm{x}}^{-1}$ & $Q_{\mathrm{E}} / Q_{\mathrm{x}}$ \\
\hline Edge (EELS) & & $(\mathrm{eV})$ & $(\mathrm{eV})$ & & $\left(\mathrm{K}_{\alpha}\right)$ & \\
\hline $\mathrm{F} \mathrm{K}$ & 9 & 685 & 8 & 0.016 & 149 & 1 \\
\hline $\mathrm{Na} \mathrm{K}$ & 11 & 1072 & 10 & 0.011 & 67 & 1 \\
\hline $\mathrm{Na} \mathrm{L} 23$ & 11 & 33 & 1 & 0.05 & 67 & 159 \\
\hline $\mathrm{P} \mathrm{L} \mathrm{L}_{23}$ & 15 & 135 & 6 & 0.03 & 20 & 90 \\
\hline $\mathrm{Cl} \mathrm{L}_{23}$ & 17 & 202 & 6 & 0.02 & 13 & 83 \\
\hline
\end{tabular}

Even though we utilize such a small fraction $\left(f_{\mathrm{E}}<0.1\right)$ of the core edge intensity for microanalysis by EELS, the useable K-shell signal is still greater than the EDXS $\mathrm{K}_{\alpha}$ signal for $Z<20$. If we can use the $L_{23}$ shell the advantage for EELS is even greater because of the much larger cross section (Fig. 1). For sodium the $\mathrm{L}_{23}$ EELS signal exceeds the $\mathrm{K}_{\alpha}$ EDXS signal by a factor of $\sim 10^{4}$.

Microanalytical sensitivity, however, is determined by the attainable $S / \sigma_{\mathrm{s}}$ (where $\sigma_{\mathrm{s}}$ is the standard deviation of the estimated signal) rather than by the signal itself. Detection limits therefore also depend strongly on the background $(B)$, particularly for EELS.

Quantitation of a characteristic EELS core edge has generally been performed by fitting the pre-edge background intensity to an inverse power law in energy and then subtracting this in the edge region [11]. As the concentration (and hence the $S / B$ ) decreases it becomes increasingly difficult to extract the signal from the background reliably. In fact we only can have confidence about the signal contained within a few $\mathrm{eV}$ of the edge, for example in a threshold resonance. The characteristic signal can then be treated like the x-ray emission "line" spectrum, the main differences being that the $S / B$ is much lower and the width of the line is much narrower. An appreciation of this similarity between the two types of spectra (EELS and EDXS) for dilute elemental concentrations has led to the use of similar processing techniques of the two spectroscopies. First or second difference filters have been used to remove the EELS background intensity in the same way that "top-hat" digital filters have been used to analyze x-ray spectra; and multiple least squares algorithms have been used to fit reference spectra in EELS as is often done in EDXS [13]. In order to estimate the relative sensitivity for EELS and EDXS we assume that the standard deviation in the estimated signal is proportional to the statistical noise $\left(N=(S+B)^{1 / 2}\right)$. If the EELS threshold 
peak and the $x$-ray line have similar shapes and similar processing techniques are used, then these constants of proportionality for EELS and EDXS are approximately equal and we obtain:

$$
\left(S / \sigma_{\mathrm{s}}\right)_{\mathrm{E}} /\left(S / \sigma_{\mathrm{s}}\right)_{\mathrm{X}} \approx(S / N)_{\mathrm{E}} /(S / N)_{\mathrm{X}}
$$

This equation is not exact because $\sigma_{\mathrm{s}}$ for EELS is increased by background removal errors, by shape differences between the unknown and reference spectra, and by detector noise. Additionally, errors reported from the MLS fit describe uncertainties over the fitting region which is not necessarily the same region used to extract the signal. Nevertheless we can estimate the relative sensitivities in terms of ratios of the signal and signal/background:

$$
\left(S / \sigma_{\mathrm{s}}\right)_{\mathrm{E}} /\left(S / \sigma_{\mathrm{s}}\right)_{\mathrm{X}} \approx\left\{\left[S_{\mathrm{E}} / S_{\mathrm{X}}\right]\left[1+(B / S)_{\mathrm{X}}\right] /\left[1+(B / S)_{\mathrm{E}}\right]\right\}^{1 / 2}
$$

If $S / B<<1$, the statistical noise is determined predominantly by the background and equation (3) can be written as,

$$
\left(S / \sigma_{\mathrm{s}}\right)_{\mathrm{E}} /\left(S / \sigma_{\mathrm{s}}\right)_{\mathrm{X}} \approx\left\{\left[S_{\mathrm{E}} / S_{\mathrm{X}}\right](S / B)_{\mathrm{E}} /(S / B)_{\mathrm{X}}\right\}^{1 / 2}
$$

Although the values of $S / B$ are quite difficult to calculate from first principles useful estimates can be readily obtained from direct measurements of simultaneously acquired EELS and EDXS spectra. Thus we can combine measured values of $(S / B)_{\mathrm{E}},(S / B)_{\mathrm{X}}$ and $S_{\mathrm{E}} / S_{\mathrm{X}}$ in order to provide a relative figure-of-merit for the two techniques. For low elemental concentrations, $(S / B)_{\mathrm{E}}$ is always small, but this is not necessarily the case for $(S / B)_{\mathrm{X}}$; therefore equation (3) rather than equation (4) is applicable.

\section{Processing of EELS.}

Spectra were recorded in the first or second difference mode $[14,15]$ in order to reduce the effects of channel-to-channel gain variations in the photodiode array. If $Y\left(E_{i}\right)$ is the intensity recorded at the $i^{\text {th }}$ channel ( $i=1$ to 1024) of the photodiode array at energy loss $E_{i}$ then the first difference spectrum, $Y^{\prime}$, is obtained by acquiring two spectra with relative energy offsets, $-\Delta / 2$ and $+\Delta / 2$, provided by a voltage applied to the drift tube of the spectrometer:

$$
Y^{\prime}\left(E_{i}\right)=Y\left(E_{i}-\Delta / 2\right)-Y\left(E_{i}+\Delta / 2\right)
$$

The second difference spectrum is obtained in a similar way by acquiring three spectra with relative energy offsets, $+\Delta, 0$, and $-\Delta$ :

$$
Y^{\prime \prime}\left(E_{i}\right)=Y\left(E_{i}\right)-0.5 Y\left(E_{i}+\Delta\right)-0.5 Y\left(E_{i}-\Delta\right)
$$

The resulting spectra, $Y^{\prime}\left(E_{i}\right)$ and $Y^{\prime \prime}\left(E_{i}\right)$, are similar to first and second derivatives respectively and the background is strongly suppressed. Furthermore, the channel-to-channel gain variations are very much smaller, although not completely eliminated. The difference acquisition technique effectively permits direct measurement of the spectrum derivatives without the amplification of the channel-to-channel gain variations that would occur if the derivatives were computed from the normal spectrum.

The energy loss spectra are quantitated by a multiple least squares fit to reference spectra. If the $k^{\text {th }}$ reference spectrum is labelled $X_{k}\left(E_{i}\right)$ where $k=1$ to $M$, then the fitting coefficients $a_{k}$ are determined by minimizing the value of $\chi^{2}$,

$$
\chi^{2}=\sum_{i=1}^{N}\left\{\left[Y\left(E_{i}\right)-\sum_{k=1}^{M} a_{k} X_{k}\left(E_{i}\right)\right]^{2} /\left[\sigma\left(E_{i}\right)\right]^{2}\right\}=\text { minimum }
$$


where $\sigma\left(E_{i}\right)$ is estimated standard error in the $i^{\text {th }}$ channel of the unknown spectrum. When the noise in the spectrum is limited by Poisson counting statistics $\left[\sigma\left(E_{i}\right)\right]^{2}$ is equal to $Y\left(E_{i}\right)$. In order to use these weights correctly the number of counts read out of the photodiode array must converted into the corresponding number of incident electrons. If we write,

$$
\begin{aligned}
\alpha_{k j} & =\sum_{i=1}^{N}\left\{\left[X_{j}\left(E_{i}\right) X_{k}\left(E_{i}\right)\right] /\left[\sigma\left(E_{i}\right)\right]^{2}\right\} \\
\beta_{k} & =\sum_{i=1}^{N}\left\{\left[Y\left(E_{i}\right) X_{k}\left(E_{i}\right)\right] /\left[\sigma\left(E_{i}\right)\right]^{2}\right\}
\end{aligned}
$$

then the solution is given by,

$$
a_{j}=\sum_{k=1}^{M}[\alpha]_{j k}^{-1} \beta_{k}
$$

and the estimated variances in coefficients $a_{j}$ are given by,

$$
\sigma^{2}\left(a_{j}\right)=[\alpha]_{j j}^{-1}
$$

The method used here for the MLS fitting is the singular value decomposition (SVD) technique [16]. Calculation of $a_{j}$ by inversion of $[\alpha]_{j k}$ is sensitive to roundoff error when fitting nearly lineardependent reference spectra. The SVD method is stable even when identical reference spectra are used, whereas the standard matrix inversion method fails completely because $[\alpha]_{j k}$ is singular.

The MLS fitting technique can be applied to first and second difference spectra as well as to the normal spectra provided the correct weights $\left[\sigma\left(E_{i}\right)\right]^{2}$ are used. These weights are derived from the normal spectra, after multiplying the photodiode counts by the conversion factor, $c$, to obtain the equivalent number of high energy electrons. For the first difference spectra the weights are $\sim 2 c Y\left(E_{i}\right)$ and for the second difference spectra $\sim 1.5 c Y\left(E_{i}\right)$. In our system the value of $c$ was found to be 40 electrons per photodiode count [2].

Changes in core edge shapes caused by plural inelastic scattering can be taken into account through inclusion of additional reference spectra; $n$ of these plural scattering terms may be obtained by successively convolving the single-scattering core edge reference spectrum with the lowloss (plasmon) spectrum $n$ times [17]. However, in this work the sample was sufficiently thin for the plural scattering to be neglected.

Small drifts in energy calibration can be also be taken into account by including an extra reference spectrum- the first derivative of the measured core edge intensity. The correction arises as the first term in a Taylor expansion of the original reference spectrum. When acquisition is performed in the first difference mode the extra term corresponds to a second derivative and a third derivative for second difference acquisition. This technique was first used to correct for small calibration variations in the analysis of $x$-ray spectra [18].

In practice it is found that the difference spectra are sometimes influenced by small intensity shifts between successive read-outs of the photodiode array. This effect can be eliminated by adding a "ramp" and "offset" as two additional reference spectra.

Quantitation of first and second difference spectra is straightforward provided the shapes of the core edges from the unknown sample region are assumed to be similar to those in the reference standards. The difference spectrum from the standard is then simply normalized by the integrated counts contained in the core edge in some arbitrary energy window, e.g. $100 \mathrm{eV}$. The coefficients of the MLS fit will thus give directly the numbers of counts in the same energy window of the 
spectrum from the unknown sample region. Quantitation can be achieved using the appropriate calculated partial ionization cross sections [11].

\section{Processing of EDXS.}

X-ray spectra were processed using the Tracor Northern BIOQ program described previously [19]. This program first passes a top-hat digital filter through the spectrum to remove the continuum background intensity. The filter width is matched to the detector resolution at the line of interest (typically 100 to $140 \mathrm{eV}$ ) and corresponds to a smoothed second derivative operation. It is also closely related to the second difference operation used for acquisition of the energy loss spectrum. The filtered spectrum is then fit by a multiple least squares procedure to a series of reference spectra that have also been filtered. The method is very similar to that described above for the energy loss spectra; estimates of errors and values of $\chi^{2}$ are also provided.

\section{Results.}

The relative sensitivities of electron energy loss and $\mathrm{x}$-ray spectroscopies for microanalysis is illustrated by a series of simultaneously recorded EELS and EDXS spectra from thin samples containing fluorine, sodium, phosphorus and chlorine (Figs. 2-5). Measurements were repeated four or five times for each sample to check consistency in the MLS error estimation.

The energy loss spectrum from octafluorostilbene [20] deposited on carbon (Fig. 2a) was recorded in the first difference mode with $\Delta=8 \mathrm{eV}$. These parameters were chosen because the fluorine $\mathrm{K}$ edge has an approximately $8 \mathrm{eV}$ width and is without a well-defined threshold peak; the first difference filter transforms an edge into a peak. The probe current was about $0.5 \mathrm{nA}$ and the acquisition time was $10 \mathrm{~s}$. A close MLS fit is found with the reference spectrum from the pure compound (bold line). The EDXS spectrum (Fig.2b) is much noisier and after an MLS analysis the advantage in $S / \sigma_{\mathrm{s}}$ for EELS over EDXS is found to be $5.7 \pm 0.8$ (Tab.II).

Table II. - Signal/background, ratio of signals and relative sensitivities for EELS and EDXS. Spectra were obtained at $100 \mathrm{keV}$ beam energy from compounds containing $\mathrm{F}, \mathrm{Na}, \mathrm{P}$ and $\mathrm{Cl}$ deposited on carbon, using Gatan 666 PEELS and Tracor Northern Micro-ZHV x-ray detector subtending 0.18 sterad solid angle. In using equation (1) to predict ratio of signals for EELS and EDXS, $\mathrm{S}_{\mathrm{E}} / \mathrm{S}_{\mathrm{X}}$ was reduced by factor of 2 for $\mathrm{F}$ and $\mathrm{Na}$ (two readouts for first difference acquisition), and factor of 3 for $\mathrm{P}$ and $\mathrm{Cl}$ (three read-outs for second difference acquisition).

\begin{tabular}{|c|c|c|c|c|c|c|}
\hline Element/ & $(S / B)_{\mathrm{E}}$ & $(S / B) \mathrm{x}$ & $S_{\mathrm{E}} / S_{\mathrm{X}}$ & $S_{\mathrm{E}} / S_{\mathrm{X}}$ & $\left(S / \sigma_{\mathrm{s}}\right)_{\mathrm{E}} /\left(S / \sigma_{\mathrm{s}}\right)_{\mathrm{X}}$ & $\left(S / \sigma_{\mathrm{s}}\right)_{\mathrm{E}} /\left(S / \sigma_{\mathrm{s}}\right)_{\mathrm{X}}$ \\
\hline Edge & meas. & meas. & meas. & $\begin{array}{l}\text { predicted } \\
\text { (Eq. 1) }\end{array}$ & $\begin{array}{l}\text { estimated } \\
\text { (Eq. 3) }\end{array}$ & from MLS \\
\hline $\mathrm{K}$ & $0.39 \pm 0.06$ & $4 \pm 1$ & $260 \pm 40$ & $85 \pm 20$ & $9.5 \pm 1.1$ & $5.7 \pm 0.8$ \\
\hline $\mathrm{Na} \quad K$ & $0.23 \pm 0.04$ & $16 \pm 4$ & $30 \pm 5$ & $25 \pm 4$ & $2.4 \pm 0.3$ & $1.1 \pm 0.2$ \\
\hline $\mathrm{Na} \quad \mathrm{L}_{23}$ & $0.17 \pm 0.03$ & $16 \pm 4$ & $15000 \pm 3000$ & $18000 \pm 3000$ & $48 \pm 9$ & $10 \pm 3$ \\
\hline $\mathrm{L}_{23}$ & $0.016 \pm 0.003$ & $3 \pm 1$ & $1200 \pm 200$ & $1100 \pm 200$ & $5.0 \pm 1.0$ & $3.6 \pm 0.5$ \\
\hline $\mathrm{Cl} \quad \mathrm{L}_{23}$ & $0.10 \pm 0.02$ & $20 \pm 5$ & $340 \pm 50$ & $500 \pm 100$ & $5.7 \pm 0.7$ & $1.4 \pm 0.3$ \\
\hline
\end{tabular}




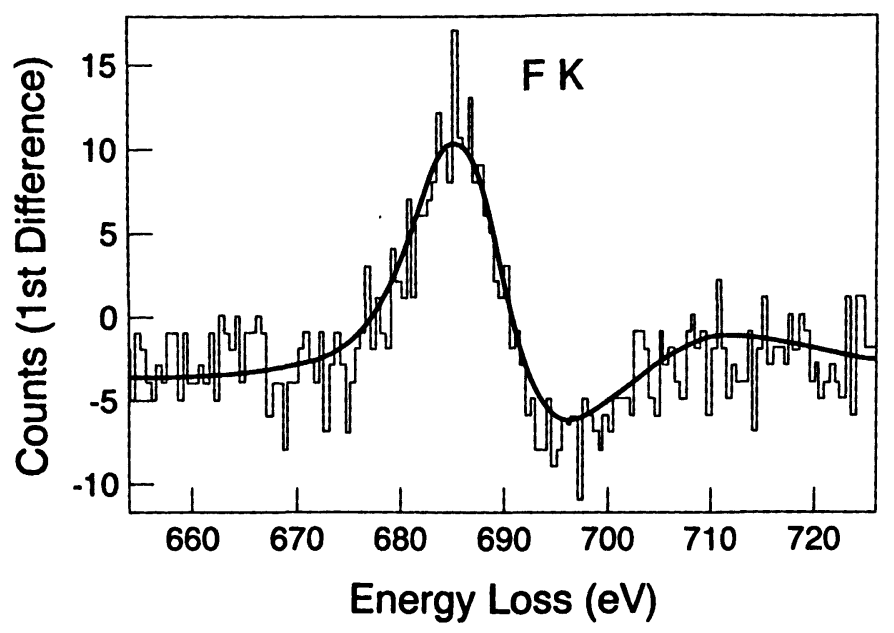

a)

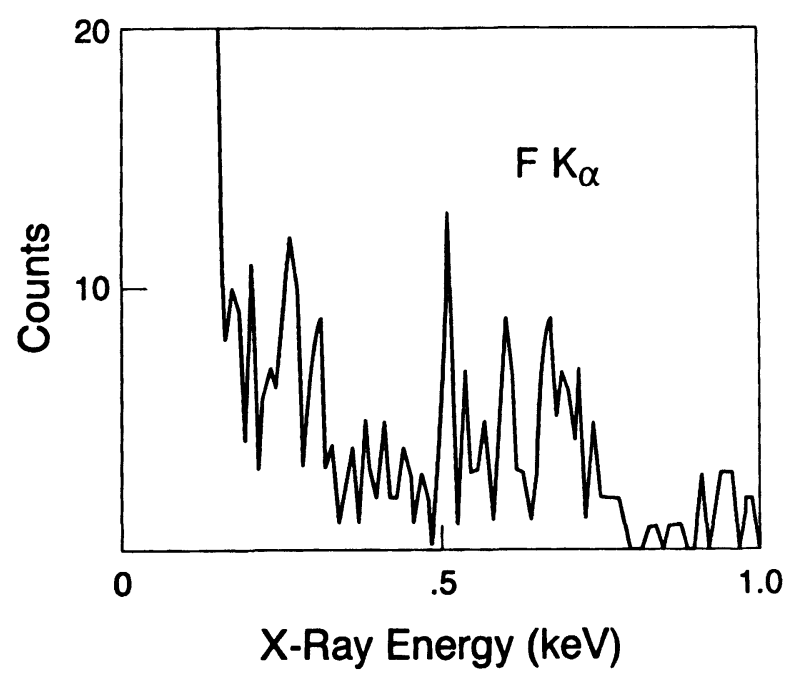

b)

Fig. 2. - (a) Fluorine K edge in first difference EELS spectrum $(\Delta=8 \mathrm{eV})$ from octafluorostilbene, acquired in 8 seconds, with MLS fit to reference spectrum (bold line). (b) simultaneously recorded EDXS spectrum.

For the sodium K edge the advantage of EELS is much smaller as is demonstrated by the spectra from $\mathrm{NaCl}$ deposited on a carbon film (Figs. $3 \mathrm{a}$ and $3 \mathrm{~b}$ ). The $\mathrm{Na} \mathrm{K}$ edge was recorded in first difference mode with $\Delta=10 \mathrm{eV}$ because of the broad edge shape. The acquisition time was $100 \mathrm{~s}$ and the probe current $\sim 0.5 \mathrm{nA}$. Again the reference spectrum fits the data satisfactorily. However, the statistics are no worse in the simultaneously acquired $\mathrm{x}$-ray spectrum; MLS analyses show that the sensitivity of EELS relative to EDXS for Na K detection is $1.1 \pm 0.2$.

If the $\mathrm{L}_{23}$ edge is used instead of the $\mathrm{K}$ edge for detection of sodium, it is interesting to find that EELS has a significant advantage over EDXS. Figures $4 \mathrm{a}$ and $4 \mathrm{~b}$ are spectra recorded from the same specimen with an acquisition time of only $1 \mathrm{~s}$. Again, the EELS spectrum is recorded in the 


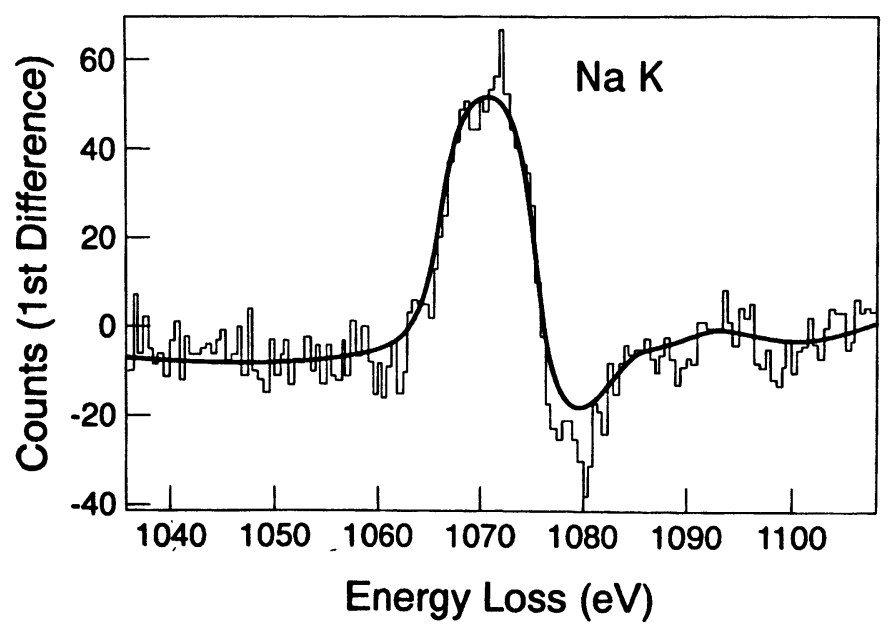

a)

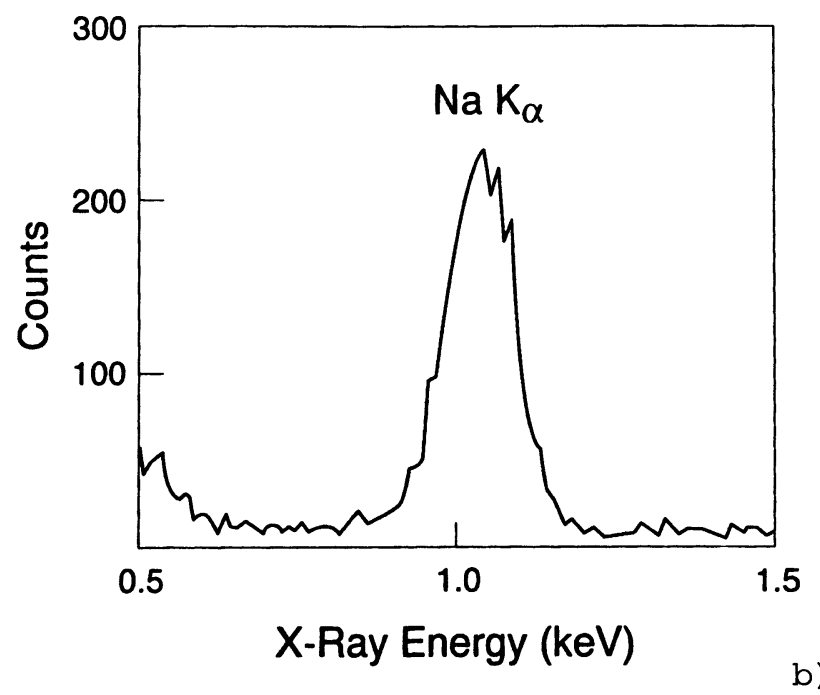

Fig. 3. - (a) Sodium K edge in first difference EELS spectrum $(\Delta=10 \mathrm{eV})$ from sodium chloride, acquired in 100 seconds, with MLS fit to reference spectrum (bold line). (b) simultaneously recorded EDXS spectrum).

first difference mode but the $\mathrm{L}_{23}$ edge at $\sim 33 \mathrm{eV}$ favors a filter width of $\Delta=1 \mathrm{eV}$. The EELS data, recorded with an energy dispersion of only $0.05 \mathrm{eV}$ per channel, are fitted satisfactorily by the reference spectrum. The statistics in the simultaneously recorded $x$-ray spectrum are now much poorer and the MLS analyses of the errors show that EELS has a factor of $10 \pm 3$ advantage in sensitivity over EDXS.

The energy loss spectrum from a thin layer of mixed sodium chloride and sodium dihydrogen phosphate salts deposited on a carbon film is shown in figure $5 \mathrm{a}\left(\mathrm{P} \mathrm{L}_{23}\right.$ edge) and figure $5 \mathrm{~b}(\mathrm{Cl}$ $\mathrm{L}_{23}$ edge). The acquisition time was $120 \mathrm{~s}$ and the probe current $\sim 0.5 \mathrm{nA}$. Shown superimposed in figures $5 \mathrm{a}$ and $5 \mathrm{~b}$ are the reference spectra obtained from pure salts which are seen to fit the 


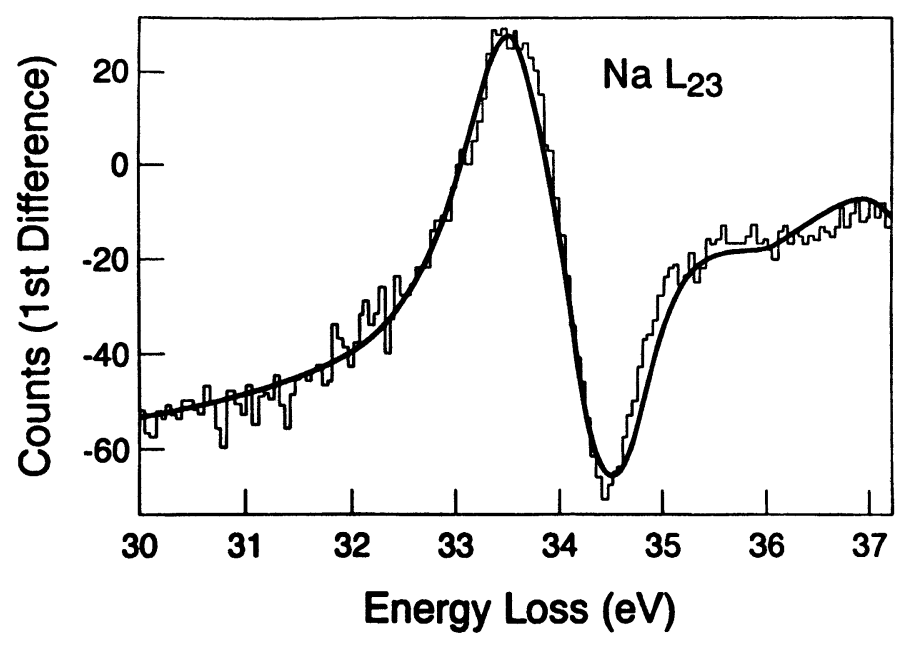

a)

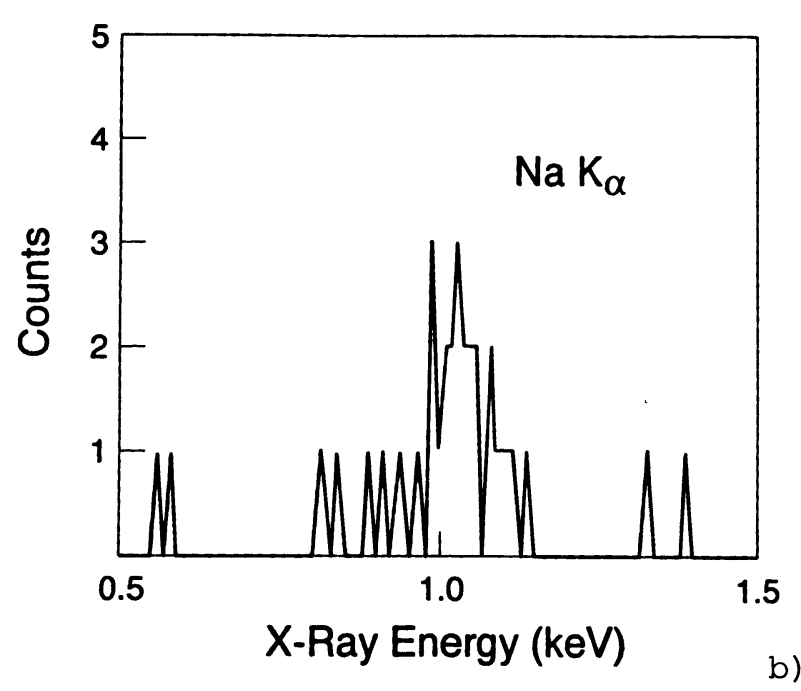

Fig. 4. - (a) Sodium $\mathrm{L}_{23}$ edge in first difference EELS spectrum $(\Delta=1 \mathrm{eV})$ from sodium chloride, acquired in 1 second, with MLS fit to reference spectrum (bold line). (b) simultaneously recorded EDXS spectrum.

data closely. The simultaneously recorded x-ray spectrum is shown in figure 5c. Estimation of the relative $S / \sigma_{\mathrm{s}}$ from the MLS analyses predicts an advantage in sensitivity of a factor of $3.6 \pm 0.5$ for phosphorus and $1.4 \pm 0.3$ for chlorine.

\section{Discussion.}

In table II we compare the experimental ratios of sensitivities for EELS and EDXS with estimates obtained from equation (3). Considering the approximations involved we find that the predicted relative sensitivities are in reasonable agreement with the results of the MLS fitting procedure. For each element the predicted sensitivity is higher than actually realized; this can be attributed 


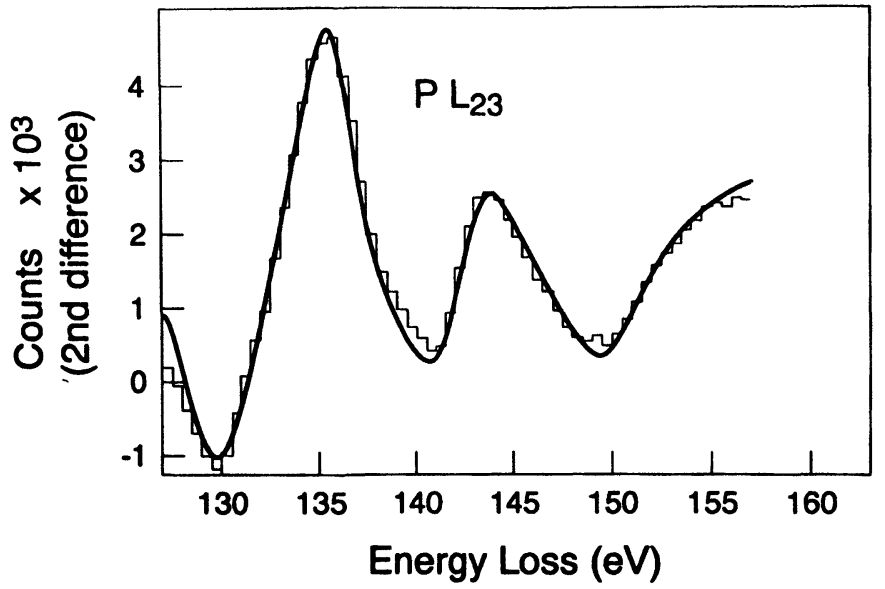

a)

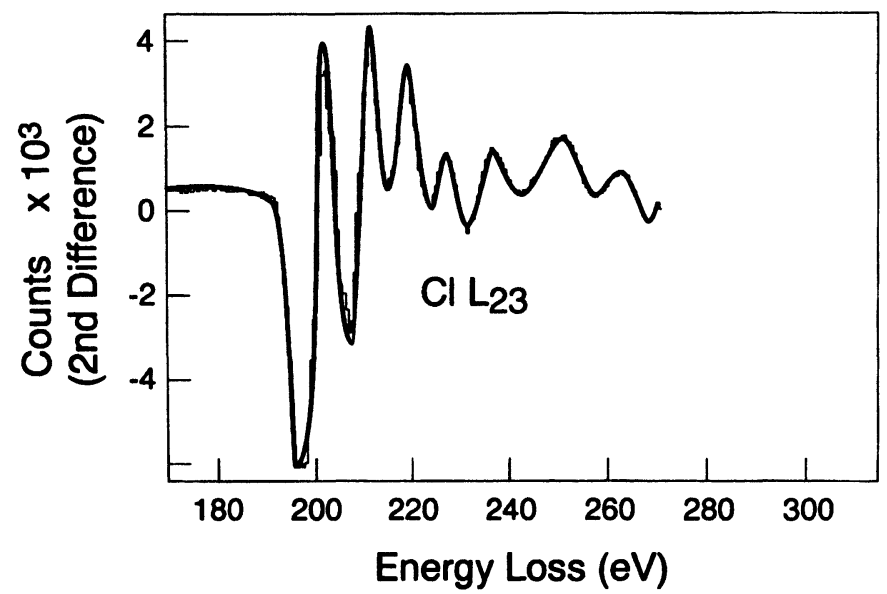

b)

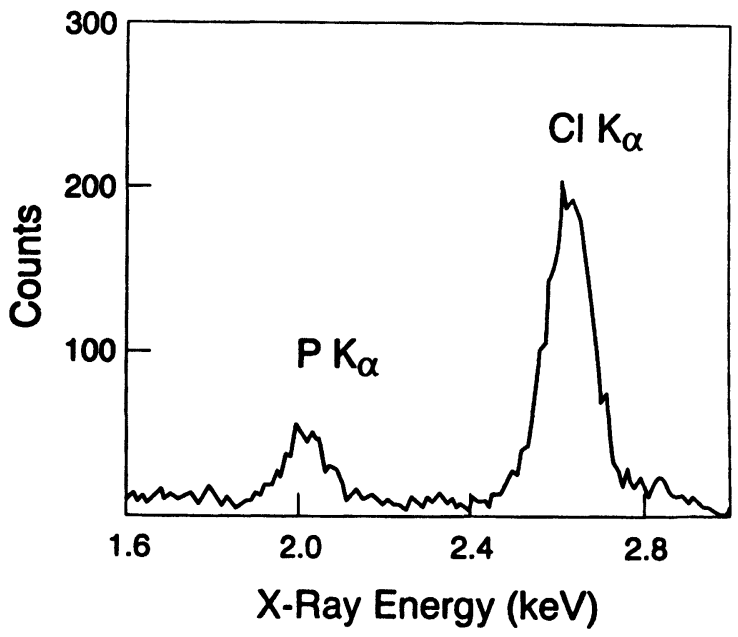

c)

Fig. 5. - (a) Phosphorus $\mathrm{L}_{23}$ edge, and (b) chlorine $\mathrm{L}_{23}$ edge in second difference EELS spectrum $(\Delta=6 \mathrm{eV})$ from mixture of sodium chloride and sodium dihydrogen phosphate, acquired in 120 seconds, with MLS fits to reference spectra (bold lines). (c) simultaneously recorded EDXS spectrum. 
to the over-simplification of equation (2). The greatest discrepancies are a factor of $\sim 4$ for the sodium and chlorine $\mathrm{L}_{23}$ edges. It should be realized, however, that equations (2-4) are intended to be applied to EELS core edges that are dominated by a single sharp peak or edge. When more complicated structure exists such as in the chlorine $L_{23}$ edge we can only expect equation (3) to provide a rough estimate. Predicted ratios of $S_{\mathrm{E}} / S_{\mathrm{X}}$ using equation (1) are generally in good agreement with the measured values indicating the validity of the parameters given in table I. Only for fluorine is the ratio of measured signals significantly different from the calculated value and this discrepancy is probably attributable to some absorption of the soft $\mathrm{F} \mathrm{K}_{\alpha} \mathrm{X}$-rays by the UTW detector.

The figure-of-merit for EELS and EDXS is also plotted as a function of atomic number in figure 6 for the situation where the elemental concentrations are very dilute. The data points (shown by crosses) are obtained from equation (4) using the experimentally determined values for $S_{\mathrm{E}} / S_{\mathrm{X}},(S / B)_{\mathrm{E}}$ and $(S / B)_{X}$. Data points for $Z=20$ (calcium) and $Z=26$ (iron) come from previously published results [21]. Continuous lines are drawn through the data to indicate the variation with atomic number and the type of atomic shell excited. From figure 6 we can see that before the EELS/EDXS sensitivity falls below unity for the $\mathrm{K}$ shell, it becomes possible to utilize the $\mathrm{L}_{23}$ shell excitation which provides a significant advantage for EELS. Then, as the EELS/EDXS sensitivity again approaches unity for the $\mathrm{L}_{23}$ edges, it is boosted by the "white-line" resonance at $Z \sim 20$. Eventually the EDXS sensitivity exceeds that of EELS near $Z=26$ (iron). At this value of $Z$, it would be interesting to determine whether the $\mathrm{M}_{23}$ edges could boost the detection limits for EELS yet again.

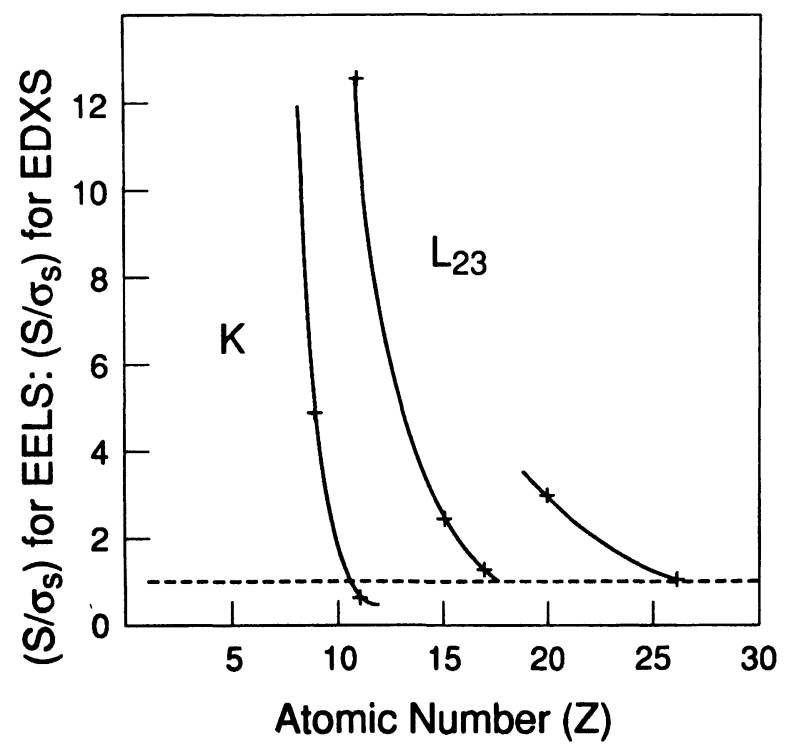

Fig. 6. - Relative sensitivities between EELS and EDXS for very dilute elements in thin carbon matrix plotted as function of atomic number $(Z)$; data points (crosses) estimated from equation (4) using measured values of $S_{\mathrm{E}} / S_{\mathrm{X}},(S / B)_{\mathrm{E}}$ and $(S / B)_{\mathrm{X}}$. Data for $\mathrm{Ca}$ and $\mathrm{Fe}$ come from earlier work [21]. Solid curves drawn to show systematic variation with $Z$. Note that $\left(S / \sigma_{\mathrm{s}}\right)_{\mathrm{E}} /\left(S / \sigma_{\mathrm{s}}\right)_{\mathrm{X}}$ is increased when elements are more concentrated because $S_{\mathrm{X}} / B_{\mathrm{X}} \gg 0$ and therefore equation (3) rather than equation (4) should be used; this situation applies to the data analyzed in Table II. 
Use of the first or second difference acquisition and MLS fitting of reference spectra for EELS quantitation has several important advantages over conventional techniques. The method is stable and is capable of extracting very small signals swamped by channel-to-channel gain variations in the detector array. Shuman $[14,15]$ has demonstrated that very small atomic concentrations can be detected $\left(\sim 10^{-4}\right)$. In addition the method is capable of separating strongly overlapping core edges where normal background subtraction techniques are inapplicable [17]. The main problem with the use of reference spectra is that the core edge shape from the unknown is assumed to be the same as that of the reference. In the examples given here this assumption is valid because the same compounds were used to generate the reference spectra. For most biological, organic or polymer samples the bonding of specific elements is often similar and so the core edge shapes do not vary much between compounds. However, for inorganic materials it will be necessary to pay close attention to the use of appropriate reference spectra. Factors such as the oxidation state, the local chemical environment, and electronic structure will all be relevant.

It is important to emphasize that the EELS and EDXS techniques are complementary. Even with a highly optimized energy loss spectrometer and optimized analysis methods many elements are best analyzed by EDXS; this is often true for $Z>30$, except for the lanthanides, actinides and a few other elements [22]. Sometimes, the lighter elements are also better analyzed by EDXS, e.g. if the sample is thicker than the inelastic mean free path, or if severe overlap occurs in the energy loss spectrum. In other situations it may be advantageous to use EELS even if EDXS sensitivity is theoretically higher. For example, there may be a "system" peak due to remote excitation of elements in the specimen environment. Iron x-rays may be generated in the objective pole-piece due to high angle scattering from the sample, or the remote excitation may be produced in the sample itself by the support grid or surrounding matrix.

\section{Conclusions.}

The experimental results, summarized in table II and figure 6, demonstrate that parallel-detection EELS has higher analytical sensitivity than EDXS for elements with $Z<17$ in a thin carbon matrix. The advantage is greatest for elements in the first row of the periodic table which are detectable by the $\mathrm{K}$ edge, and for elements at the beginning of the next row which are detectable by the $\mathrm{L}_{23}$ edge. We have attempted to provide a quantitative basis for assessing the relative advantages of EELS and EDXS. Provided care is taken to obtain suitable reference spectra, our approach could be used to compare sensitivities of the techniques for analysis of elements in metallic, semiconductor or ceramic matrices. This would result in a new set of curves, similar to those presented here, and provide a useful guide for the analyst deciding the best strategy to solve a particular problem.

\section{Acknowledgements.}

One of the authors (J.A.H.) would like to acknowledge support from the National Science Foundation grant\# DMR8905459. 


\section{References}

[1] SHUMAN H., Ultramicroscopy, 6 (1981) 163-168.

[2] KRIVANEK O.L., AHN C.C. and KEENEY R.B., Ultramicroscopy 22 (1987) 103-116.

[3] Statham P.J., J. Phys. France 45 (1984) C2-175.

[4] THOMAS L.E., Ultramicroscopy 18 (1985) 173-184.

[5] KRIVANEK O.L., MORY C., TENCE M. and COLLIEX C. (to be published).

[6 ISAACSON M.S. and JOHNSON D., Ultramicroscopy 1 (1975) 33-52.

[7] KRIVANEK O.L., PATERSON J.H. and POPPA H.R., In: Proceedings of the 47th Annual Meeting of EMSA, G.W. Bailey Ed. (San Francisco Press, San Francisco, 1989) 410-411.

[8] McCARTHY J.J., In: Proceedings of the 46th Annual Meeting of EMSA G.W. Bailey Ed. (San Francisco Press, San Francisco, 1988) 670-671.

[9] Kundmann M., Chabert X., Truong K. and KrivaneK O. (1990) EL/P software for Macintosh II computer, Gatan Inc., Pleasanton, CA 94566, USA.

[10] HUNT J., In: Proceedings of the 47th Annual Meeting of EMSA, G.W. Bailey Ed. (San Francsico Press, San Francisco, 1989) 398-399.

[11] EgERTON R.F., In: Electron Energy Loss Spectroscopy in the Electron Microscope (Plenum, New York, 1986).

[12] HEINRICH K.F.J., In: Electron beam x-ray microanalysis (Van Nostrand Reinhold, New York, 1981).

[13] SHUMAN H., SOMLYO A.V. and SOMLYO A.P., Ultramicroscopy 1 (1976) 317-339.

[14] SHUMAN H. and KRUIT P., Rev. Sci. Instrum. 56 (1985) 231-239.

[15] SHUMAN H. and SOMLYO A.P., Ultramicroscopy 21 (1987) 23-32.

[16] Press W.H., FlanNERY B.P., TEukOlSKy S.A. and VeTteRling W.T., Numerical Recipes in C: The Art of Scientific Computing (Cambridge University Press, 1988) pp. 534-539.

[17] LEAPMAN R.D. and SWYT C.R., Ultramicroscopy 26 (1988) 393-404.

[18] KiTAZAWA T., SHUMAN H. and SOMLYO A.P., Ultramicroscopy 11 (1983) 251-262.

[19] SCHAMBER F.H., In: Proc. 8th Natl. Conf. on Electron Probe Microanalysis (1973) 85A-C.

[20] CiliaX B.J., KIRK K.L. and Leapman R.D., In: Proc. 12 Internat. Cong. Electron Microscopy, Seattle L.D. Peachey, D.B. Williams Eds. (San Francisco Press, San Francisco, CA) 2 (1990) 408-409.

[21] LEAPMAN R.D., In: Electron Probe Microanalysis- Applications in Biology and Medicine, K. Zierold and H.K. Hagler (Eds.), Springer Series in Biophysics, (Springer-Verlag, Berlin) 4 (1989) 113-125.

[22] AHN C.C. and KRIVANEK O.L., In: EELS Atlas, ASU Center for Solid State Science, Tempe, AZ; and Gatan Inc., Warrendale, PA (1983). 